\title{
Mobile Robot with Image Recognition -- Using LabVIEW and KNRm
}

\author{
Kuo-Hsien Hsia \\ Bachelor Program in Interdisciplinary Studies \\ National Yunlin University of Science and Technology \\ 123 University Road, Section 3, Douliou, Yunlin 64002, Taiwan, R.O.C. \\ Bo-Jung Yang \\ Graduate School of Engineering Science and Technology \\ National Yunlin University of Science and Technology \\ 123 University Road, Section 3, Douliou, Yunlin 64002, Taiwan, R.O.C. \\ Jr-Hung Guo \\ Bachelor Program in Interdisciplinary Studies \\ National Yunlin University of Science and Technology \\ 123 University Road, Section 3, Douliou, Yunlin 64002, Taiwan, R.O.C. \\ Chang-Sheng Xiao \\ Department of Electrical Engineering \\ National Yunlin University of Science and Technology \\ 123 University Road, Section 3, Douliou, Yunlin 64002, Taiwan, R.O.C. \\ E-mail: \{khhsia,D10810211,jrhung,M10712003\}@yuntech.edu.tw \\ www.yuntech.edu.tw
}

\begin{abstract}
The main purpose of this paper is to use the image recognition of LabVIEW software to construct mobile robots with various functions, and make the robots applicable to the industry and have web monitoring applications. The core of the robot is mainly the KNRm controller. This controller is suitable for beginners, and can be connected to DC servo motor, RC servo motor, infrared, ultrasonic and camera to achieve various functions of the robot. The structure of the robot uses metal parts sold by Studica company, which can be in accordance with the desired function to assemble the robot. Since the company is a designated equipment sponsor company for World Skills competitions, it can also be in line with international standards. Finally, PID control and sensors are added to make the robot movement and position more accurately.
\end{abstract}

Keywords: Image Recognition, Mobile Robot, Web monitoring, KNRm.

\section{Introduction}

With the increasing advancement of science and technology, the products of robots are becoming more and more diversified, especially in terms of mobile robots. Mobile robots are generally closely related to people's lives, such as sweeping robots and robots used in restaurants. Many countries are focused on the robot industry. And the growth of mobile robots will be faster than industrial robots. The design and development of mobile robots has also received increasing attention in the field of education and competition $[1,2,3]$. Taking the high school industrial student skills competition held by the Ministry of Education, which is the most important

(C) The 2021 International Conference on Artificial Life and Robotics (ICAROB2021), January 21 to 24, 2021 
high school competition in Taiwan, as an example, the number of participants has grown from 86 to 102 in 3 years [4]. For Taiwan, which is facing a declining birthrate, this is a big growth rate. This shows the booming development of robot education.

\section{Problem Description}

This paper takes the project of the 49th National Skills Competition in Taiwan as the subject of problem solving. The competitors are asked to design a toy organizing robot. This robot can help the children put the toys into categories after the game. The layout of the competition field is shown in Fig. 1. Zone A in Fig. 1 is the starting area of the robot, and the size of the robot must be completely within the range of the starting area. There is a height-limit gate on the passage as shown in Fig. 1 marked as G, and the robot must be below this height to pass the gate. Zone $\mathrm{B}$ is the golf ball sprinkling area. Before the start of the evaluation, 5 to 6 golf balls of 5 colors are sprinkled via the sprinkler, as shown in Fig. 2, to Zone $\mathrm{B}$. Zone $\mathrm{C}$ is the target ball-collection area. There are five slots in Zone C. A barcode is hanged on the wall of each grid to indicate balls of what color should be placed in the grid. At the beginning, the robot starts from the starting area, and firstly reads the barcode of each grid in Zone $\mathrm{C}$, and then passes through the passage and gate to the golf sprinkling area to pick up the golf ball. After gripping, the robot returns to the ball-collection area and places the balls according to the color assigned by the barcode. The robot must collect the balls as much as possible and place them to the correct slots within the limited time duration, and return to the departure area before the end of the time. In order to test the design of the robot mechanism under limited resources, the number of motors and sensors are limited as mentioned in the test project.

\section{Hardware Design}

We mainly use the integrated kit, including Tetrix metal blocks, sensors and motors packed by Studica, to design and assemble the robot according to the assigned functions. As for the controller, the KNRm developed by KKITC is used, and its main core is NI myRIO-1950. The main difference from $\mathrm{KNRm}$ to myRIO is that a single myRIO needs to be wired by oneself or a suitable expansion board needs to be made by oneself, and the

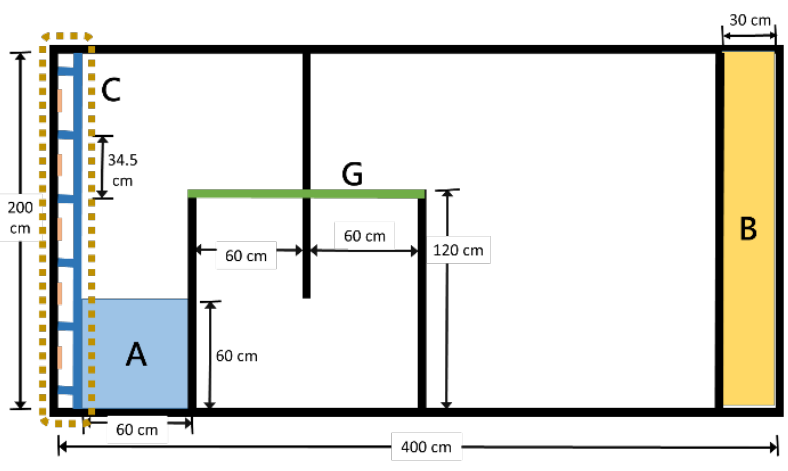

Fig. 1. Field of the considered problem.

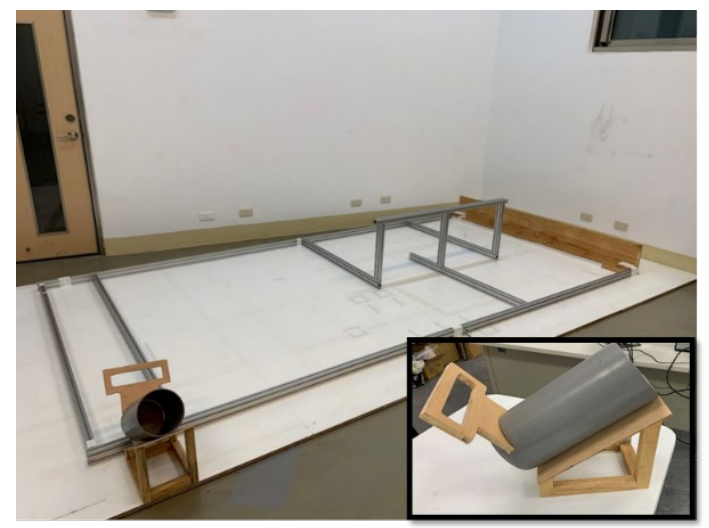

Fig. 2. The field with the sprinkler.

useful pins can be wired out. Most of the pins that need to be used in $\mathrm{KNRm}$ have been designed around the controller for user convenience. Like myRIO, KNRm can be programmed with LabVIEW, developed by National Instruments (NI) Company. Different from traditional programming language, LabVIEW uses $\mathrm{G}$ language, a data-flow design, that allows users to programming according to the desired functions in conceptualization. In addition, $G$ language has the ability of parallel processing [5].

In terms of mechanism design, because the golf balls have to be gripped out from the sprinkling area, a lifting mechanism and a ball clamping mechanism are necessary. In addition, the picked golf balls need to be stored on the robot. Hence a ball storage mechanism is required. It requires the use of $3 \mathrm{RC}$ servo motors to meet above requirements, so an RC expansion box must be used. Due to the use of the RC expansion box will occupy one DC motor-port and there are only 4 motor-ports in a KNRm, only 3 DC motors with omni wheels can be used for the chassis. With omni wheels, it can achieve the functions 
of forward, backward, left and right motion, rotation and diagonal movement of the chassis.

The analysis about the robot body design is as following.

1. The ball clamping mechanism: Since the golf balls have to be moved to the collection area in a limited time, the design of the clamp mechanism is to clamp as many golf balls as possible. However, the blocks provided by Studica are not suitable for use. We design a customized part by 3D printing.

2. The ball storage mechanism: Due to the need of storing multiple golf balls on the robot without increasing the number of RC motors, a ramp with end-block is designed to allow the balls to roll into for storage.

3. The lifting mechanism: In order for the balls rolling smoothly into the ball storage mechanism, it is necessary to raise the clamp mechanism to a certain height. In addition, beside the lifting mechanism, a mechanism that can move back and forth must also be connected.

The overall design of the mechanism of the robot is shown in Fig. 3.

For obstacle avoidance, we use ultrasonic sensors as the main sensor, and infrared sensors as an auxiliary. The reason is to improve the accuracy of travel and overcome the lack of infrared rays that can pass directly through when encountering certain obstacles. The advantage of ultrasound is that the distance is farther than infrared. The ultrasonic sensor used in this paper is PING))), and the infrared sensor used is SHARP GP2Y0A21YK0F.

In the motion control part of the robot, we use trapezoidal acceleration and deceleration and PID control for control.

\section{Image Recognition}

In the problem to be solved in this paper, barcode reading and color determination are needed. Therefore, we use Logitech C310 camera as the image sensing device. In LabVIEW, users can combine their own image processing programs with built-in VIs, or use the Vision Assistant function of LabVIEW to achieve quick start processing. Vision Assistant is an integrated program for LabVIEW image processing, which has many image processing functions. The Vision Acquisition program can be used to set up the camera, including Acquisition Source, Acquisition Type, camera resolution and frame

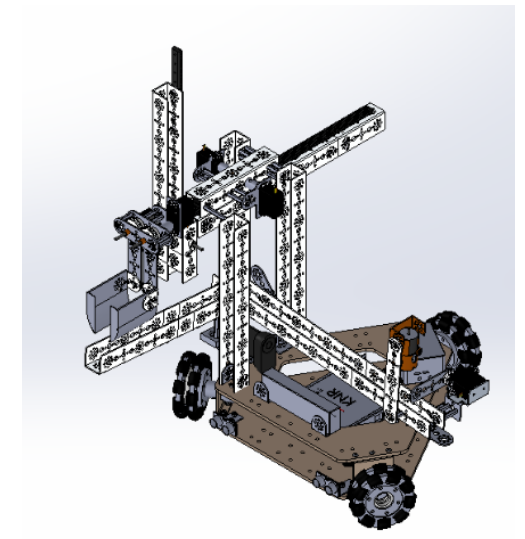

Fig. 3. The mechanism of the designed robot.

number, etc. Then use Vision Assistant to process the image obtained by the camera. In Vision Assistant, one can set and process the options of Image, Color, Grayscale, Binary, Machine vision, Identification and other items. Because the images we deal with in this paper are mainly the interpretation and recognition of bar codes and golf balls, and bar code interpretation is a builtin function in LabVIEW, we use Find Circular Edge and color comparison directly instead of the technique of image gray-scale processing in the paper.

\section{Experimental Results}

We conducted field test with the robots we designed. After the robot leaves the starting area, it reads the barcodes first, as shown in Fig. 4, and stores the color specified for each ball slot. After that, the height of the robot must be lowered so that it can pass through the gates in the passage smoothly. After the robot has completely passed the passage, it uses the side wall to correct the robot's posture, and turns to make the gripper face to the golf sprinkling area, and then goes to the sprinkling area. In order to facilitate the gripping of golf balls scattered in the sprinkling area, the robot will sweep the balls into the same area after reaching the sprinkling area, and then perform the action of gripping the balls. After performing multiple clamping, the robot then returns according to the path it came. When passing through the gate, the clamping mechanism should be lowered to pass it. After passing through the gates, the gripper returns to the original position, and place the golf ball in the corresponding grid when the robot passes

(C) The 2021 International Conference on Artificial Life and Robotics (ICAROB2021), January 21 to 24, 2021 
through each collection area. Finally, after releasing all the balls, the robot returns to the starting area to complete the experiment. After actual testing, the robot we designed can be successfully used to achieve the tasks required by the problem and return to the starting area, as shown in Fig. 5.

\section{Discussions and Conclusions}

In this paper, a KNRm is used as the core controller of the robot, combined with the Tetrix metal building blocks, three motors, three RC servo motors and appropriate sensing devices, to design a solution that can solve the project of Mobile Robotics of 49th National Skills Competition in Taiwan. The designed robot has been verified on the field and found that it can indeed achieve the specified task.

Because it is composed of metal building blocks, the designed robot will be insufficient in terms of mechanical rigidity. When designing robots to solve other application problems, metal building blocks similar to this paper can also be used to design the prototype of the robot to test whether the function can meet the requirements or not. When the designed robot can meet the requirements, one can proceed with the appearance design of the robot to be used as a commercial product. At the same time, the mechanical rigidity of the robot is included in the material consideration. This can greatly reduce the time for product development, and can more accurately demand for application products.

\section{References}

1. S.E. Jung and E.S. Won, Systematic Review of Research Trends in Robotics Education for Young Children, Sustainability 10(4), 2018, 905

2. D. Karahoca, A. Karahoca, H. Uzunboylu, Robotics Teaching in Primary School Education by Project based Learning for Supporting Science and Technology Courses, Procedia Computer Science 3, 2011, pp. 1425-1431.

3. D. Bazylev, A. Margun, K. Zimenko, A. Kremlev, E. Rukujzha, Participation in robotics competition as motivation for learning, Procedia - Social and Behavioral Sciences 152, 2014, pp. 835-840.

4. National High Schools Skills Competition. (in Chinese) Available: http://sci.me.ntnu.edu.tw/Contest/ Retrieved: 11/30/2012.

5. C.H. Tseng, W.H. Guan, W.H.Wu, Advanced Teaching Manual of Robot with LabVIEW - Building Robots with

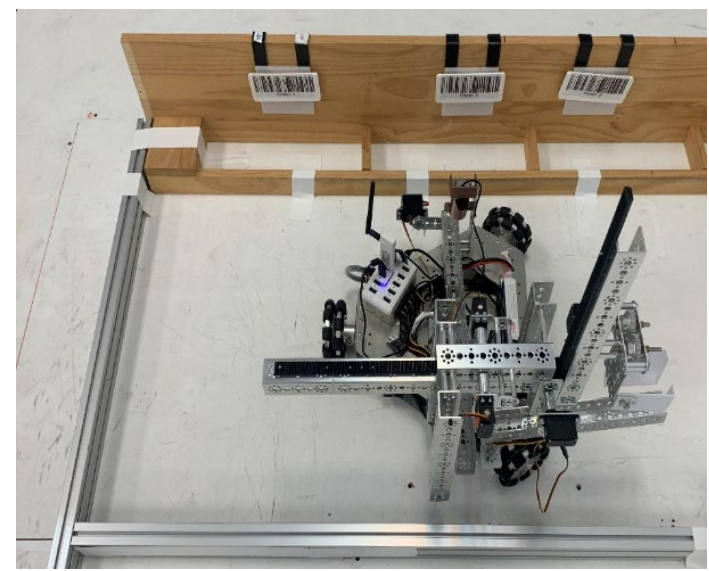

Fig. 4. The robot starts and reads the barcodes.

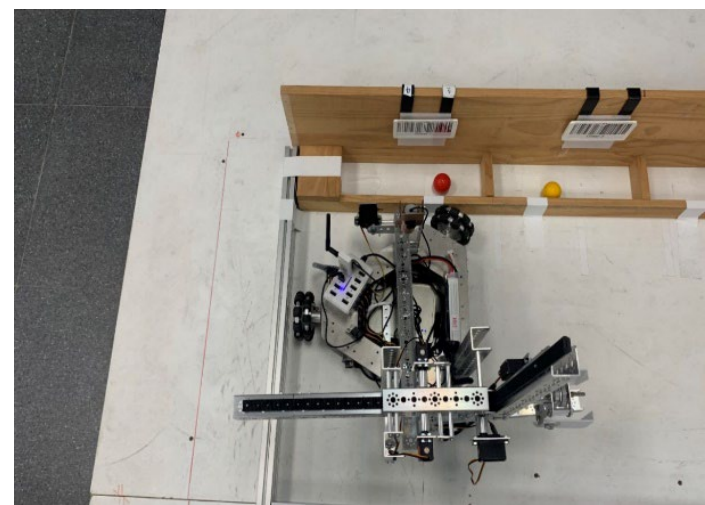

Fig. 5. Task completed.

Both Intelligence and Interest, 2nd edn., GOTOP Press, Taipei, 2014. (in Chinese) 